\title{
Method of self-consistent evaluation of absolute emission probabilities of particles and gamma rays
}

\author{
Sergei Badikov ${ }^{1, a}$ and Valery Chechev $^{2}$ \\ 1 National Research Nuclear University "MePHI”, 127287 Moscow, Russia \\ 2 V.G. Khlopin Radium Institute, 194021 St. Petersburg, Russia
}

\begin{abstract}
In assumption of well installed decay scheme the method provides a) exact balance relationships, b) lower (compared to the traditional techniques) uncertainties of recommended absolute emission probabilities of particles and gamma rays, c) evaluation of correlations between the recommended emission probabilities (for the same and different decay modes). Application of the method for the decay data evaluation for even curium isotopes led to paradoxical results. The multidimensional confidence regions for the probabilities of the most intensive alpha transitions constructed on the basis of present and the ENDF/BVII.1, JEFF-3.1, DDEP evaluations are inconsistent whereas the confidence intervals for the evaluated probabilities of single transitions agree with each other.
\end{abstract}

\section{Introduction}

This study was basically motivated by the following reasons. As known most of decay schemes are not exactly balanced. As an example in Table 1 sums of absolute emission probabilities for particles and gammarays feeding the states and emitted from the states of daughter nucleus in decay of ${ }^{244} \mathrm{Cm}$ are presented (DDEP evaluation [1]). These values are strongly disbalanced for 4 excited states.

The results of the evaluation must also meet the metrology requirements. GUM [2] defines the results of the multidimensional evaluation as complete if those contain three types of the data: vector of estimates, its covariance matrix and the confidence region. None of existing decay data evaluation methods provides such information for a set of the evaluated absolute emission probabilities.

These reasons stimulated the development of the decay data evaluation method providing the exact balance relationships and a calculation of the covariances between the evaluated decay data.

This study is also of interest from the theoretical point of view as an example of the analysis of multiparticle closed system.

\section{Statistical model and basic equations}

The problem of the evaluation of absolute emission probabilities of particles and gamma-rays with taking exact balance relationships into account can be reduced to a recurrent solution of a linear system of equations

$$
\mathbf{X} \theta+\varepsilon=\mathbf{y}
$$

a e-mail: sabadikov@mephi.ru with $m$ restrictions imposed to the vector $\theta$ of $L$ parameters being evaluated

$$
\mathbf{H} \theta=\mathbf{d}
$$

Here $\mathbf{y}$ and $\boldsymbol{\varepsilon}$ are the vectors (of dimension $n>L$ ) of measurements and unbiased random experimental errors, $\mathbf{X}$ is the matrix of sensitivity coefficients of the model function relative to the parameters

$$
X_{i j}=\partial f\left(E_{i}, \theta\right) / \partial \theta_{j},
$$

$\mathbf{H}$ is a matrix of dimension $m \times L$, $\mathbf{d}$ is a vector of dimension $m$. The elements of the matrix $\mathbf{H}$ and the vector $\mathbf{d}$ are known. Type of the model function is determined by the physics of the problem

$$
f(E \theta)=\sum_{i=1}^{L} \theta_{i} \varphi_{i}(E),
$$

where functions $\varphi_{1}(E), \ldots, \varphi_{L}(E)$ are defined on a discrete set of energies $E_{1}, \ldots, E_{L}$ in the following way

$$
\varphi_{i}\left(E_{j}\right)=\delta_{i j}= \begin{cases}1 & i=j \\ 0 & i \neq j\end{cases}
$$

The evaluation problem defined by Eqs. (1)-(5) can be solved by a minimization of the functional

$$
\tilde{S}^{2}(\vec{\theta}, \vec{\lambda})=(\vec{y}-X \vec{\theta})^{T} \mathbf{V}^{-1}(\vec{y}-\mathbf{X} \vec{\theta})+\vec{\lambda}^{T}(\mathbf{H} \vec{\theta}-\vec{d})
$$

where $\vec{\lambda}^{T}=\left(\lambda_{1}, \ldots, \lambda_{m}\right)$ are the Lagrange multipliers. The functional (6) gets a minimum at

$$
\tilde{\vec{\theta}}=\hat{\vec{\theta}}+\mathbf{W H}^{T}\left(\mathbf{H W H} \mathbf{H}^{T}\right)^{-1}(\vec{d}-\mathbf{H} \hat{\vec{\theta}}),
$$

with a covariance matrix

$$
\mathbf{U}=\mathbf{W}-\mathbf{W H}^{T}\left(\mathbf{H W H} \mathbf{H}^{T}\right)^{-1} \mathbf{H W} .
$$

(C) The Authors, published by EDP Sciences. This is an Open Access article distributed under the terms of the Creative Commons Attribution License 4.0 (http://creativecommons.org/licenses/by/4.0/). 
Table 1. Level balance checking in decay of ${ }^{244} \mathrm{Cm}$.

\begin{tabular}{|l|c|c|c|c|}
\hline Level & $\begin{array}{c}\text { Level } \\
\text { energy,keV }\end{array}$ & Sum feeding & Sum emitting & $\begin{array}{c}\text { Relative } \\
\text { error, \% }\end{array}$ \\
\hline 1 & 42.824 & 23.3 & 23.4 & 0.324 \\
\hline 2 & 141.69 & 0.0240 & 0.0239 & 0.305 \\
\hline 3 & 294.32 & 0.00356 & 0.00355 & 0.281 \\
\hline 4 & 497.60 & 0.0000400 & 0.0000400 & 0 \\
\hline 5 & 597.34 & 0.0001416 & 0.0001420 & 0.2817 \\
\hline 6 & 648.85 & 0.0000211 & 0.0000169 & 24.9 \\
\hline 7 & 860.71 & 0.000149 & 0.000134 & 11.2 \\
\hline 8 & 900.32 & 0.0000500 & 0.0000530 & 5.66 \\
\hline 9 & 938.06 & 0.00000470 & 0.00000890 & 47.2 \\
\hline
\end{tabular}

Table 2. The evaluated ${ }^{242} \mathrm{Cm}$ and ${ }^{244} \mathrm{Cm}$ alpha transition probabilities and their uncertainties compared to the DDEP, ENDF/B-VII.1 and the JEFF-3.1 evaluations (per 100 decays).

\begin{tabular}{|l|c|c|c|c|c|}
\hline $\mathbf{N}$ & $\mathbf{E}_{\alpha}, \mathbf{k e V}$ & this work & DDEP & ENDF/B-VII.1 & JEFF-3.1 \\
\hline $0\left({ }^{242} \mathrm{Cm}\right)$ & 6113 & $74.04(4)$ & $74.06(7)$ & $74.08(7)$ & $74.0(9)$ \\
\hline $1\left({ }^{242} \mathrm{Cm}\right)$ & 6069 & $25.92(4)$ & $25.94(7)$ & $25.92(6)$ & $26.0(9)$ \\
\hline $2\left({ }^{242} \mathrm{Cm}\right)$ & 5969 & $0.034(1)$ & $0.034(2)$ & $0.035(2)$ & $0.0350(1)$ \\
\hline $0\left({ }^{244} \mathrm{Cm}\right)$ & 5805 & $76.76(10)$ & $76.7(4)$ & $76.9(1)$ & $76.6(1)$ \\
\hline $1\left({ }^{244} \mathrm{Cm}\right)$ & 5763 & $23.21(10)$ & $23.3(4)$ & $23.1(1)$ & $23.4(1)$ \\
\hline $2\left({ }^{244} \mathrm{Cm}\right)$ & 5665 & $0.0204(6)$ & $0.0204(15)$ & $0.0204(15)$ & $0.027(3)$ \\
\hline
\end{tabular}

The right part of the expressions (7) and (8) consists of 2 terms. The first terms represent the solution of the classical least squares problem (LSP) and its covariance matrix. The second terms are the corrections to the LSP solution and its covariance matrix induced by the inclusion of the restriction (2) into evaluation process. The schemes of calculations can be organized as indicated in $[3,4]$.

\section{Results of calculations}

The examples of an application of the method for the ${ }^{242} \mathrm{Cm}$ and ${ }^{244} \mathrm{Cm}$ decay data evaluation are given in Refs. $[3,4]$. In Table 2 the ${ }^{242} \mathrm{Cm}$ and ${ }^{244} \mathrm{Cm}$ alpha emission intensities (for most strong transitions) calculated with the method of interest are presented compared to the ENDF/BVII.1, JEFF-3.1 and DDEP evaluations.

At first glance, all the evaluated data for the ${ }^{244} \mathrm{Cm}$ $(0,0)$ and $(0,1)$ alpha transitions are consistent within declared uncertainties. However, a consideration of uncertainties of the recommended data for the multidimensional evaluations (carried out separately from the correlations) leads to incorrect interpretation of the evaluated results. An adequate interpretation of the multidimensional evaluations can be obtained by a construction of the confidence regions for each of the evaluations. In Fig. 1 the confidence regions of the $(0,0)$ and $(0,1){ }^{244} \mathrm{Cm}$ alpha emission probabilities corresponding to present evaluation and the ENDF/B-VII.1, JEFF-3.1 and DDEP evaluations are shown. As seen from Fig. 1 a correct conclusion is following: the present evaluation is inconsistent with other evaluations since these evaluations don't take the strength of weak alpha transitions into account. At the same time making the projections of the confidence regions to $\mathrm{x}$ - and $y$-axis we can conclude that all the evaluations for the single $(0,0)$ and $(0,1)$ alpha transitions are consistent.

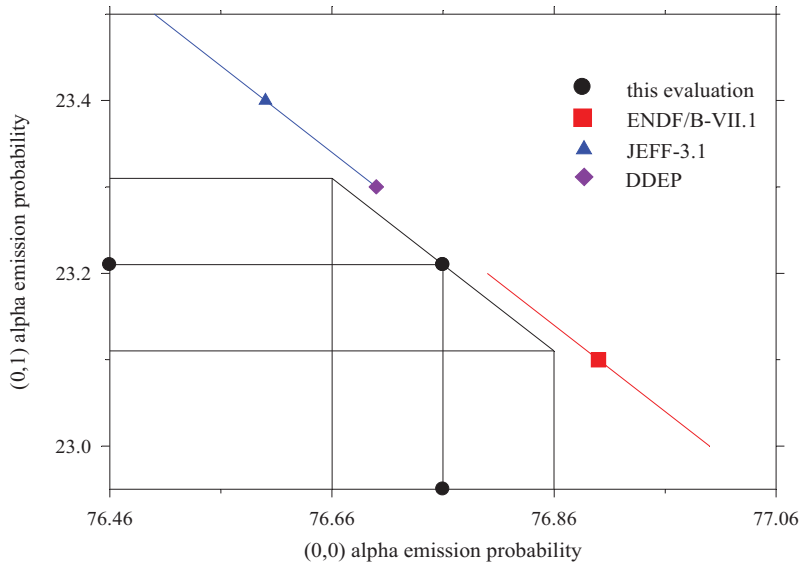

Figure 1. Confidence regions (solid lines) of evaluated $(0,0)$ and $(0,1)$ alpha emission probabilities in decay of $\mathrm{Cm}-244$ from this work and the ENDF/B-VII.1, JEFF-3.1 evaluations. The uncertainties of the emission probabilities are shown for present evaluation.

\section{Summary}

The basic features of the method of self-consistent evaluation are as follows.

A decay process is considered as a transformation of a closed system from one state to another one. The closure of the system is provided by taking exact balance relationships into account.

Unlike other techniques the method handles the parameters being evaluated as a multidimensional vector.

The self-consistency of the evaluated data is provided by applying the iterative procedure in calculations.

The inclusion of exact balance relationships in the evaluation process leads to significantly lower uncertainties of the evaluated data (compared to the traditional methods) and strong correlations between some of the evaluated parameters.

Application of the method for the decay data evaluation of even curium isotopes leads to paradoxical results. The two-dimensional confidence regions for the probabilities of intense alpha transitions constructed on the basis of present and the ENDF/B-VII.1, JEFF-3.1, DDEP evaluations are inconsistent whereas the confidence intervals for the evaluated probabilities of single transitions agree with each other. The effect is explained by taking exact balance relationships into account in present evaluation.

\section{References}

[1] M.-M. Bé, V. Chisté, C. Dulieu, X. Mougeot, V.P. Chechev, F.G. Kondev, A.L. Nichols, X. Huang, B. Wang, 2013. Table of Radionuclides (Vol. 7 $\mathrm{A}=14$ to 245) and Comments on evaluations, Monographie BIPM-5, Bureau International des Poids Et Mesures, Pavillon de Breteuil, 92312 Sèvres, France and http://www. nucleide.org/NucData.htm

[2] Supplement 2 to the "Guide to the expression of uncertainty in measurement" - Extension to any number of output quantities, JCGM 102 (2011)

[3] S.A. Badikov, V.P. Chechev, Applied Radiation and Isotopes 87, 137 (2014)

[4] S.A. Badikov, V.P. Chechev, Applied Radiation and Isotopes 109, 164 (2016) 